\title{
A note on projective norm graphs
}

\author{
Codruţ Grosu* \\ grosu.codrut@gmail.com
}

\begin{abstract}
The projective norm graphs $\mathcal{P}(q, 4)$ introduced by Alon, Rónyai and Szabó are explicit examples of extremal graphs not containing $K_{4,7}$. Ball and Pepe showed that $\mathcal{P}(q, 4)$ does not contain a copy of $K_{5,5}$ either for $q \geq 7$, asymptotically improving the best lower bound for $\operatorname{ex}\left(n, K_{5,5}\right)$.

We show that these results can not be improved, in the sense that $\mathcal{P}(q, 4)$ contains a copy of $K_{4,6}$ for infinitely many primes $q$.
\end{abstract}

\section{Introduction}

Let $H$ be any graph. For any $n \geq 1$, the Turán function $\operatorname{ex}(n, H)$ is defined as the maximum possible number of edges of an $H$-free graph on $n$ vertices. Although the precise value of the Turán function is known in several cases, for arbitrary graphs $H$ the best result we have is an asymptotic estimate provided by the Erdős-Stone-Simonovits theorem:

$$
\operatorname{ex}(n, H)=\left(1-\frac{1}{\chi(H)-1}+o(1)\right)\left(\begin{array}{l}
n \\
2
\end{array}\right)
$$

where $\chi(H)$ is the chromatic number of $H$. This estimate is particularly poor when $H$ is bipartite $(\chi(H)=2)$, as then it only tells us that $\operatorname{ex}(n, H)=o\left(n^{2}\right)$. Therefore it makes sense to try to obtain better estimates for bipartite graphs.

Even the case of complete bipartite graphs $K_{t, s}$ is not fully solved. Kővári, Sós and Turán (11]) showed that $\operatorname{ex}\left(n, K_{t, s}\right) \leq c_{t, s} n^{2-\frac{1}{t}}$ for any $s \geq t$, where $c_{t, s}>0$ is a constant depending only on $t$ and $s$. While the upper bound is believed to be tight, the best general lower bound, obtained by the probabilistic method $(\underline{8})$, only gives

$$
\operatorname{ex}\left(n, K_{t, s}\right) \geq c n^{2-\frac{s+t-2}{s t-1}} .
$$

Nevertheless, the upper bound is tight for $t=2$, as shown by Erdös, Rényi and Sós (7 $)$, and $t=3$, as shown by Brown (4). On the other hand, the asymptotic behaviour of ex $\left(n, K_{t, t}\right)$ is not known for any $t \geq 4$.

A significant step towards a solution to this problem was made by Kollár, Rónyai and Szabó ([10]), who constructed the norm graphs $\mathcal{G}(q, t)$, a family of graphs which are extremal for $K_{t, s}$,

${ }^{*}$ This research was supported by the Deutsche Forschungsgemeinschaft within the research training group 'Methods for Discrete Structures' (GRK 1408). 
for any $s \geq t !+1$. This construction was further refined by Alon, Rónyai and Szabó ([1]), who introduced the projective norm graphs $\mathcal{P}(q, t)$, graphs which are extremal for $K_{t, s}$, for any $s \geq(t-1) !+1$.

The construction of $\mathcal{P}(q, t)$ is as follows. Let $t \geq 3$ and $q$ be a prime power. We let $\mathbb{F}_{q}$ be the finite field with $q$ elements.

We define $\mathcal{P}(q, t)$ as the graph on vertex set $\mathbb{F}_{q^{t-1}} \times \mathbb{F}_{q}^{*}$, with an edge between two vertices $(\alpha, a)$ and $(\beta, b)$ if and only if $N(\alpha+\beta)=a b$. Here $N(x)$ denotes the norm of the element $x$ relative to the extension $\mathbb{F}_{q^{t-1}} / \mathbb{F}_{q}$. Then the following holds.

Theorem 1 (1]). Any $t$ distinct vertices in $\mathcal{P}(q, t)$ have at most $(t-1)$ ! common neighbors.

Later several other families of graphs avoiding large bipartite subgraphs were constructed ([3], [5]). However, none of these constructions matches (or improves) the bound $s \geq(t-1) !+1$, and in fact to this day the projective norm graphs remain the best construction we have: for no $s \leq(t-1)$ ! and $t$ large enough do we know an example of a graph improving (2).

For $t=4, \mathcal{P}(q, 4)$ avoids $K_{4,7}$. It was shown by Ball and Pepe ([2]) that for $q \geq 7, \mathcal{P}(q, 4)$ further avoids $K_{5,5}$, thus improving the lower bound on $\operatorname{ex}\left(n, K_{5,5}\right)$ to $\frac{1}{2}(1+o(1)) n^{7 / 4}$. As the best lower bound on $\operatorname{ex}\left(n, K_{4,4}\right)$ is

$$
\operatorname{ex}\left(n, K_{4,4}\right) \geq \frac{1}{2} n^{5 / 3}+o\left(n^{5 / 3}\right)
$$

following from Brown's optimal construction for $K_{3,3}$, it would be desirable to know whether $\mathcal{P}(q, 4)$ further avoids any of the subgraphs $K_{4,4}, K_{4,5}$ or $K_{4,6}$. The main result of this note is the following.

Theorem 2. There exists an infinite sequence of primes of density $\frac{1}{9}$ such that for any prime $p$ in this sequence, $\mathcal{P}(p, 4)$ contains a copy of $K_{4,6}$.

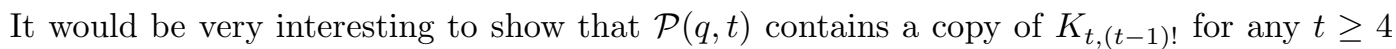
and infinitely many $q$. I strongly believe that this general claim should have a beautiful simple proof; but so far I have not found any.

As an amend to this, I show the following.

Theorem 3. For any $t \geq 4$ and any $m \geq 1$, there exists an infinite sequence of primes of positive density such that for any prime $p$ in this sequence, $\mathcal{P}(p, t)$ contains a copy of $K_{t-1, m}$.

Of course it follows directly from the Kövári-Sós-Turán theorem that $\mathcal{P}(q, t)$ contains arbitrarily large $K_{t-1, m}$. The point of Theorem 3 is that it provides an explicit construction of such subgraphs.

The proof of Theorem 2 is of the following nature. A relatively well-behaved copy of $K_{4,6}$ is found in $\mathcal{P}(7,4)$ using a computer search. Then using the approach of [15] and [9], this copy is lifted to infinitely many primes $p$. However, the techniques in 9 only apply if the initial copy is in a finite field of very large characteristic. It is rather remarkable that the same methods can be used if the starting prime is small (in our case, 7). Furthermore, an additional theoretical complication is introduced by the use of the norm; this requires a more delicate handling of the Galois groups involved.

\section{$2 \quad$ A Galois extension of $\mathbb{Q}$}

The plan to prove Theorem 2 is the following. We shall first define two finite extensions $E / \mathbb{Q}, F / E$ such that $[F: E]=3$. Then in $F / E \times E$ we will construct a copy of $K_{4,6}$, with the norm condition fulfilled by $N_{F / E}(x)$. We will then show that $E$ maps in a suitable sense to $\mathbb{F}_{p}$, for infinitely many $p$, while $F$ maps to a degree 3 extension of $\mathbb{F}_{p}$. As the norm essentially stays the same, this will give a copy of $K_{4,6}$ in $\mathcal{P}(p, 4)$. 
Let $\zeta$ be a third root of unity. Let $\theta_{1}$ be the root $\sqrt[3]{2}$ of the polynomial $x^{3}-2$ and $\theta_{2}$ the root $\sqrt[3]{3}$ of the polynomial $x^{3}-3$. Define $F:=\mathbb{Q}\left(\zeta, \theta_{2}, \theta_{1}\right)$.

Lemma 4. The field $F$ is a Galois extension of $\mathbb{Q}$ of degree $[F: \mathbb{Q}]=18$. A basis of $F / \mathbb{Q}$ is given by all elements of the form $\zeta^{i} \theta_{1}^{j} \theta_{2}^{k}$, with $0 \leq i \leq 1$ and $0 \leq j, k \leq 2$.

Proof. The roots of the polynomial $x^{3}-2$ are $\zeta^{i} \theta_{1}$ with $0 \leq i \leq 2$. Similarly, the roots of the polynomial $x^{3}-3$ are $\zeta^{i} \theta_{2}, 0 \leq i \leq 2$. Hence $F$ is the splitting field of $\left(x^{3}-2\right)\left(x^{3}-3\right)$, in particular a Galois extension of $\mathbb{Q}$.

Clearly $[\mathbb{Q}(\zeta): \mathbb{Q}]=2$ with basis $\{1, \zeta\}$, and $[F: \mathbb{Q}(\zeta)] \leq 9$. We show that the elements of $A:=\left\{\theta_{1}^{j} \theta_{2}^{k}: 0 \leq j, k \leq 2\right\}$ are pairwise linearly independent over $\mathbb{Q}(\zeta)$. Indeed, if

$$
\alpha \theta_{1}^{j_{1}} \theta_{2}^{k_{1}}+\beta \theta_{1}^{j_{2}} \theta_{2}^{k_{2}}=0, \quad \alpha, \beta \in \mathbb{Q}(\zeta)^{*},\left(j_{1}, k_{1}\right) \neq\left(j_{2}, k_{2}\right),
$$

then $\theta_{1}^{j_{1}-j_{2}} \theta_{2}^{k_{1}-k_{2}} \in \mathbb{Q}(\zeta)$. Then $\mathbb{Q}(\zeta)$ contains a cube root of $2,3,6$ or 12 , and hence has degree at least 3 over $\mathbb{Q}$, a contradiction.

Then by Theorem 1.3, 6], $A$ is linearly independent over $\mathbb{Q}(\zeta)$. Hence $F / \mathbb{Q}(\zeta)$ is an extension of degree 9 and has $A$ as a basis. Then the elements $\zeta^{i} \theta_{1}^{j} \theta_{2}^{k}$ also form a basis of $F$ over $\mathbb{Q}$.

Let $S:=\left\{\zeta^{i} \theta_{1}^{j} \theta_{2}^{k}: 0 \leq i \leq 1,0 \leq j, k \leq 2\right\}$ be the basis of $F / \mathbb{Q}$.

Let $G$ be the Galois group of $F / \mathbb{Q}$. Any element $\tau \in G$ is described by how it acts on $\zeta, \theta_{1}$ and $\theta_{2}$. Consider the maps

$$
\tau_{1}: \zeta \rightarrow \zeta^{2}, \quad \tau_{2}: \theta_{1} \rightarrow \zeta \theta_{1}, \quad \tau_{3}: \theta_{2} \rightarrow \zeta \theta_{2},
$$

which act on one element of $\left\{\zeta, \theta_{1}, \theta_{2}\right\}$ and fix the remaining two. This defines $\tau_{i}$ at every element of $S$, and by linearity we can extend each $\tau_{i}$ to the whole of $F$. Then $\tau_{i}$ is multiplicative on $S$, in particular $\tau_{i} \in G$. It follows that $\tau_{1}, \tau_{2}$ and $\tau_{3}$ generate $G$ (as they generate 18 distinct elements). But the subgroup $\left\langle\tau_{2}, \tau_{3}>\right.$ is isomorphic to $\mathbb{Z}_{3} \times \mathbb{Z}_{3}$, a direct product of two cyclic groups, and $\tau_{1}$ acts as conjugation. Thus $G$ is isomorphic to the semi-direct product $\left(\mathbb{Z}_{3} \times \mathbb{Z}_{3}\right) \rtimes_{\phi} \mathbb{Z}_{2}$, with the map $\phi: \mathbb{Z}_{3} \times \mathbb{Z}_{3} \rightarrow \mathbb{Z}_{3} \times \mathbb{Z}_{3}$ sending an element to its inverse.

Let $\sigma \in G$ be the Galois automorphism sending $\zeta \rightarrow \zeta, \theta_{1} \rightarrow \zeta \theta_{1}$ and $\theta_{2} \rightarrow \zeta^{2} \theta_{2}$. Then $\sigma$ corresponds to the element $(1,2) \rtimes_{\phi} 0$, and so has conjugacy class $C_{\sigma}=\left\{\sigma, \sigma^{2}\right\}$.

Note that $\left\{1, \sigma, \sigma^{2}\right\}$ is a subgroup of $G$. Let $E \subset F$ be the fixed field of this subgroup. Then $\mathbb{Q}(\zeta) \subset E$. However, as $\sigma$ permutes the roots of $x^{3}-2$ cyclically, this polynomial is still irreducible over $E$. Then $F=E\left(\theta_{1}\right)$. The fields $E$ and $F$ are our desired extensions.

\section{The construction of $K_{4,6}$}

We are now ready to describe the construction of $K_{4,6}$ in $F / E \times E$. We shall use the basis $1, \theta_{1}, \theta_{1}^{2}$ of $F / E$.

Consider the polynomial $g(x):=x^{3}+21 x^{2}+3 x+7$. It has the roots

$$
-4 \cdot 6^{1 / 3}-2 \cdot 6^{2 / 3}-7, \quad-4 \cdot 6^{1 / 3} \zeta-2 \cdot 6^{2 / 3} \bar{\zeta}-7, \quad-4 \cdot 6^{1 / 3} \bar{\zeta}-2 \cdot 6^{2 / 3} \zeta-7,
$$

which all lie in $F$. The most important thing for us is that $\sigma\left(6^{1 / 3}\right)=\sigma\left(\theta_{1} \theta_{2}\right)=\zeta^{3} \theta_{1} \theta_{2}=6^{1 / 3}$, and hence $\sigma$ fixes all the roots of $g$. Then all the roots of $g$ belong to $E$.

Now define $A:=\left\{(0,3),(1,4),(2,5),\left(\theta_{1}+1,6\right)\right\}$. Further define

$$
\begin{aligned}
B:=\left\{\left(\zeta^{k} \theta_{1}^{2}-1,1\right): 0 \leq k \leq 2\right\} & \\
& \bigcup\left\{\left(-\frac{1-\eta}{4} \theta_{1}^{2}-\frac{1+\eta}{2} \theta_{1}-1, \frac{1+3 \eta^{2}}{4}\right): \eta \text { a root of } g\right\} .
\end{aligned}
$$

Any element of $A \cup B$ is of the form $(\alpha, a)$ with $\alpha \in F$ and $a \in E$. The existence of a $K_{4,6}$ is shown by the following. 
Lemma 5. For any $(\alpha, u) \in A$ and any $(\beta, v) \in B$ we have $N_{F / E}(\alpha+\beta)=u v$.

Proof. Let $x:=a \theta_{1}^{2}+b \theta_{1}+c, a, b, c \in E$, be any element of $F$. The norm $N_{F / E}(x)$ is defined as the determinant of the linear map $y \rightarrow x y$. For the basis $1, \theta_{1}, \theta_{1}^{2}$ we get

$$
N_{F / E}(x)=\operatorname{det}\left(\begin{array}{ccc}
c & 2 a & 2 b \\
b & c & 2 a \\
a & b & c
\end{array}\right)=c^{3}+2 b^{3}+4 a^{3}-6 a b c
$$

By our choice of $B, \beta$ is of the form $a \theta_{1}^{2}+b \theta_{1}-1$. Thus we need to check that the following holds:

$$
\begin{aligned}
N_{F / E}\left(a \theta_{1}^{2}+b \theta_{1}-1\right) & =4 a^{3}+2 b^{3}+6 a b-1 & & =3 v, \\
N_{F / E}\left(a \theta_{1}^{2}+b \theta_{1}\right) & =4 a^{3}+2 b^{3} & & =4 v, \\
N_{F / E}\left(a \theta_{1}^{2}+b \theta_{1}+1\right) & =4 a^{3}+2 b^{3}-6 a b+1 & & =5 v \\
N_{F / E}\left(a \theta_{1}^{2}+(b+1) \theta_{1}\right) & =4 a^{3}+2 b^{3}+6 b^{2}+6 b+2 & & =6 v,
\end{aligned}
$$

for any $(\beta, v) \in B$. This readily follows if $(\beta, v)$ is of the form $\left(\zeta^{k} \theta_{1}^{2}-1,1\right)$.

So assume $(\beta, v)=\left(-\frac{1-\eta}{4} \theta_{1}^{2}-\frac{1+\eta}{2} \theta_{1}-1, \frac{1+3 \eta^{2}}{4}\right)$ for some root $\eta$ of $g$.

As $\eta^{3}=-21 \eta^{2}-3 \eta-7$, we have $(1-\eta)^{3}=8+24 \eta^{2}$. Also $(1+\eta)^{3}=-6-18 \eta^{2}$. Consequently

$$
4\left(-\frac{1-\eta}{4}\right)^{3}+2\left(-\frac{1+\eta}{2}\right)^{3}=-\frac{1}{16}\left(8+24 \eta^{2}\right)+\frac{1}{4}\left(6+18 \eta^{2}\right)=1+3 \eta^{2}=4 v
$$

Furthermore,

$$
1-6\left(-\frac{1-\eta}{4}\right)\left(-\frac{1+\eta}{2}\right)=1-\frac{3}{4}\left(1-\eta^{2}\right)=v .
$$

These two facts together verify the first three norm equations. For the final one, note that

$$
3\left(-\frac{1+\eta}{2}\right)^{2}+3\left(-\frac{1+\eta}{2}\right)+1=\frac{3+3 \eta^{2}+6 \eta}{4}-\frac{3+3 \eta}{2}+1=\frac{1+3 \eta^{2}}{4}=v
$$

This implies that the last norm equation holds as well, finishing the proof.

For the proof of Theorem 2 we need one further ingredient.

Theorem 6 (Chebotarëv's density theorem, [14]). Let $f$ be a polynomial with integer coefficients and with leading coefficient 1 . Assume that the discriminant $\Delta(f)$ of $f$ does not vanish. Let $C$ be a conjugacy class of the Galois group $G$ of $f$. Then the set of primes $p$ not dividing $\Delta(f)$ for which the Frobenius substitution $\sigma_{p}$ belongs to $C$ has a density, and this density equals $\frac{|C|}{|G|}$.

In what follows we shall use the terminology from 14. In particular, if $K$ is a field of characteristic 0 and $p$ a prime number, a place of $K$ over $p$ is a map $\psi: K \rightarrow \overline{\mathbb{F}}_{p} \cup\{\infty\}$ with the following properties:

(i) $\psi^{-1} \overline{\mathbb{F}}_{p}$ is a subring of $K$, and $\psi: \psi^{-1} \overline{\mathbb{F}}_{p} \rightarrow \overline{\mathbb{F}}_{p}$ is a ring homomorphism;

(ii) $\psi(x)=\infty$ if and only if $\psi\left(x^{-1}\right)=0$, for any non-zero $x \in K$.

See [12], Chapter 8, for a standard treatment of Chebotarëv's theorem. 
Proof of Theorem 娄. The polynomial $f(x):=\left(x^{3}-2\right)\left(x^{3}-3\right)$ has discriminant $\Delta(f)=26244$ and splitting field $F$. Applying Theorem 6 to the conjugacy class $C_{\sigma}$ of $\sigma$ in the Galois group $G$ of $f$ gives an infinite sequence $\mathcal{S}$ of primes of density $\frac{\left|C_{\sigma}\right|}{|G|}=\frac{1}{9}$.

Let $p$ be any prime in $\mathcal{S}$ not dividing the discriminant $\Delta(g)=-248832$ of $g$, and $\psi$ a place of $F$ over $p$. By assumption, $\sigma_{p} \in C_{\sigma}=\left\{\sigma, \sigma^{2}\right\}$. Thus the Frobenius automorphism of $\mathbb{F}_{p}$ fixes the elements of $\psi(E)$, and permutes cyclically the roots of $x^{3}-2$.

Hence $\psi$ maps any root of $g$ to an element of $\mathbb{F}_{p} \cup\{\infty\}$. However, as $x^{3} g\left(\frac{1}{x}\right)$ has constant term 1, none of the roots of $g$ map to $\infty$. As $p \nmid \backslash \Delta(g)$, all the roots of $g$ are distinct elements of $\mathbb{F}_{p}$.

For the same reason there is a cube root of unity present in $\mathbb{F}_{p}$.

As the roots of $x^{3}-2$ are permuted cyclically, $x^{3}-2$ stays irreducible over $\mathbb{F}_{p}$. Let us adjoin the root $\psi\left(\theta_{1}\right)$ of $x^{3}-2$ to form $\mathbb{F}_{p^{3}}$. Then the sets $\psi(A)$ and $\psi(B)$ are well-defined in $\mathbb{F}_{p^{3}} / \mathbb{F}_{p}$. Furthermore $\psi\left(N_{F / E}(x)\right)=N_{\mathbb{F}_{p^{3}} / \mathbb{F}_{p}}(\psi(x))$ holds for any $x=\alpha+\beta$, with $(\alpha, a) \in A,(\beta, b) \in B$. Then Lemma 5 shows that $\psi(A)$ and $\psi(B)$ form a $K_{4,6}$ in $\mathcal{P}(p, 4)$.

\section{The construction of $K_{t-1, m}$}

In this section we prove Theorem 3 Let $t \geq 4$ and $m \geq 1$ be arbitrary. Let $\theta_{1}, \theta_{2}, \ldots, \theta_{m}$ be the roots of $x^{m}-2$. This time there is no particular reason for choosing this polynomial; any $m$ conjugates will do. Let $L$ be the splitting field of $\left(x^{m}-2\right)\left(x^{t-2}-1\right)$.

Define the polynomials $f_{i}(x)=x^{t-1}-x+\theta_{i}, 1 \leq i \leq m$. The derivative $f_{1}^{\prime}(x)=(t-1) x^{t-2}-1$ has $t-2$ distinct roots $x_{1}, \ldots, x_{t-2}$. Then

$$
f_{1}\left(x_{i}\right)=\frac{x_{i}}{t-1}-x_{i}+\theta_{1}=-\left(1-\frac{1}{t-1}\right) x_{i}+\theta_{1} .
$$

Hence $f_{1}$ takes distinct values at $x_{i}, 1 \leq i \leq t-2$. We now use the following theorem of Hilbert.

Theorem 7 ([13], Theorem 4.4.5). Let $K$ be any field of characteristic 0 , and $g \in K[x]$ of degree $n$. Suppose $g^{\prime}(x)$ has distinct zeroes, and $g$ takes distinct values at the zeroes of $g^{\prime}$. Then $g(x)-T$ has Galois group $S_{n}$ over $K(T)$.

Therefore $f_{1}(x)-T$ is irreducible and with Galois group $S_{t-1}$ over $L(T)$. By Hilbert Irreducibility (13], Theorem 3.4.1 and Proposition 3.3.5), we can find an integer $r$ such that $f_{1}(x)-r$ stays irreducible over $L$ and still has Galois group $S_{t-1}$.

Let $F$ be the splitting field of $f_{1}(x)-r$ over $L$. Let $\sigma$ be the Galois automorphism of $F$ corresponding to the element $(12 \ldots t-1)$ in the Galois group of $F / L$. Then $\sigma$ generates a subgroup $H=\left\{1, \sigma, \ldots, \sigma^{t-2}\right\}$. Let $E:=F^{H}$ be the fixed field of this subgroup.

Lemma 8. Let $\theta_{i} \in E$ be any conjugate of $\theta_{1}$. Then $\sigma$ permutes the roots of the polynomial $f_{i}(x)-r=x^{t-1}-x+\theta_{i}-r$ cyclically, for any $1 \leq i \leq m$.

Proof. Take a $\mathbb{Q}$-automorphism of $L$ mapping $\theta_{1}$ to $\theta_{i}$, and extend it to an automorphism $\tau$ of $F$ mapping $E$ to itself.

As $f_{1}(x)-r$ splits over $F$, so does $f_{i}(x)-r=\tau\left(f_{1}(x)-r\right)$. Hence if $\sigma$ does not permute the roots of $f_{i}(x)-r$ cyclically, then $f_{i}(x)-r$ factors as $g_{i}(x) h_{i}(x)$ over $E$. It follows that $f_{1}(x)-r=\tau^{-1}\left(f_{i}(x)-r\right)=\tau^{-1}\left(g_{i}(x)\right) \tau^{-1}\left(h_{i}(x)\right)$, and so $f_{1}(x)-r$ also factors over $E$, a contradiction with the fact that $f_{1}(x)-r$ is irreducible over $E$.

Now apply Theorem 6 to the minimal polynomial of a primitive element in $F / \mathbb{Q}$ and the conjugacy class of $\sigma$ in $\operatorname{Gal}(F / \mathbb{Q})$, to obtain a sequence of primes of positive density. Let $p$ be any prime in this sequence, and $\psi$ a place of $F$ over $p$. Then $\psi$ maps all the roots $\theta_{i}$ to elements of $\mathbb{F}_{p}$. By design, $\psi\left(f_{1}(x)-r\right)$ is an irreducible polynomial of degree $t-1$ over $\mathbb{F}_{p}$ : adjoin some 
root to obtain $\mathbb{F}_{p^{t-1}}$. By Lemma 8, all the polynomials $\psi\left(f_{i}(x)-r\right)$ are irreducible over $\mathbb{F}_{p}$, and split in $\mathbb{F}_{p^{t-1}}$. Let $\alpha_{i}$ be a root of the polynomial $\psi\left(f_{i}(x)-r\right), 1 \leq i \leq m$. Further let $\zeta$ be a $(t-2)$-root of unity, which must belong to $\mathbb{F}_{p}$ by construction.

Now define $A:=\left\{\left(\zeta^{k}, 1\right): 1 \leq k \leq t-2\right\} \cup\{(0,1)\}$.

Also let $B:=\left\{\left(-\alpha_{i}, \psi\left(\theta_{i}-r\right)\right): 1 \leq i \leq m\right\}$.

Then $|A|=t-1,|B|=m$ and $A \cup B$ are vertices of $\mathcal{P}(p, t)$. We claim that they form a $K_{t-1, m}$ in $\mathcal{P}(p, t)$. To see this, note that for any $1 \leq k \leq t-2$ and $1 \leq i \leq m$,

$$
N_{\mathbb{F}_{p^{t-1}} / \mathbb{F}_{p}}\left(\zeta^{k}+\left(-\alpha_{i}\right)\right)=\psi\left(f_{i}-r\right)\left(\zeta^{k}\right)=\psi\left(\theta_{i}-r\right) .
$$

Furthermore,

$$
N_{\mathbb{F}_{p^{t-1} / \mathbb{F}_{p}}}\left(0+\left(-\alpha_{i}\right)\right)=\psi\left(f_{i}-r\right)(0)=\psi\left(\theta_{i}-r\right)
$$

as well. This shows that $A \cup B$ indeed defines a $K_{t-1, m}$, as desired.

Acknowledgements. The question answered in this paper was raised during the Discrete Mathematics III seminar held at Freie Universität Berlin in the winter of 2015. I would like to thank all the participants of the seminar for their motivating talks.

\section{References}

[1] N. Alon, L. Rónyai, and T. Szabó. Norm-graphs: Variations and Applications. J. Comb. Theory Ser. B, 76(2):280-290, 1999.

[2] S. Ball and V. Pepe. Asymptotic improvements to the lower bound of certain bipartite Turán numbers. Combin. Probab. Comput., 21:323-329, 2012.

[3] P. Blagojević, B. Bukh, and R. Karasev. Turán numbers for $K_{s, t}$-free graphs: topological obstructions and algebraic constructions. Israel J. Math., 197(1):199-214, 2013.

[4] W. G. Brown. On graphs that do not contain a Thomsen graph. Canad. Math. Bull., 9:281-289, 1966.

[5] B. Bukh. Random algebraic construction of extremal graphs. Bull. London Math. Soc., 47(6):939-945, 2015.

[6] R. Carr and C. O'Sullivan. On the Linear Independence of Roots. Int. J. Number Theory, $5(1): 161-171,2009$.

[7] P. Erdős, A. Rényi, and V. T. Sós. On a problem of graph theory. Studia. Sci. Math. Hungar., 1:215-235, 1966.

[8] P. Erdős and J. Spencer. Probabilistic Methods in Combinatorics. Akadémiai Kiadó, Budapest, 1974.

[9] C. Grosu. $\mathbb{F}_{p}$ is locally like C. J. London Math. Soc., 89(3):724-744, 2014.

[10] J. Kollár, L. Rónyai, and T. Szabó. Norm-graphs and Bipartite Turán Numbers. Combinatorica, 16(3):399-406, 1996.

[11] T. Kővári, V. T. Sós, and P. Turán. On a problem of K. Zarankiewicz. Colloq. Math., 3:50-57, 1954.

[12] J. S. Milne. Algebraic Number Theory.http://www.jmilne.org/math/CourseNotes/ant.html

[13] J. P. Serre. Topics in Galois Theory. Jones and Bartlett, Boston, 1992.

[14] P. Stevenhagen and H. W. Lenstra. Chebotarëv and his density theorem. Math. Intelligencer, 18(2):26-37, 1996.

[15] V. H. Vu, M. M. Wood, and P. M. Wood. Mapping incidences. J. London Math. Soc., 84(2):433-445, 2011. 